\title{
Thoughts on RFM scoring
}

Received (in revised form): 22nd May, 2000

\section{John R. Miglautsch}

founded and serves as chairman of Miglautsch Marketing, Inc. which provides database marketing systems and consultation to direct marketing clients across North America and the EU. Their Model-to-Mail ${ }^{\mathrm{TM}}$ system allows ordinary marketing people to increase profits dramatically by building and mailing with statistical models at their desk in only a few hours. John is the founder of The International Society for Strategic Marketing Ltd, a think tank for direct marketing strategy. At present, members hail from 67 countries.

\begin{abstract}
Direct marketing is fundamentally the scientific control of customer acquisition and contact. This paper addresses the fundamental questions of RFM (recency, frequency and monetary). Since direct marketing segmentation is a science, it is important to quantify customer behaviour so that the short- and long-term effect of the segmentation formulae can be tested. The purpose of RFM is to provide a simple framework for quantifying customer behaviour. Too often, direct marketers will use static customer selections. This paper explains clearly why RFM is a superior method for selecting customers.
\end{abstract}

\section{RFM BASICS}

Direct marketing is fundamentally the scientific control of customer acquisition and contact. The fundamental question is whether customer A merits an additional contact based on their past purchase behaviour. This question applies equally to direct mail, catalogue, phone, field or Internet contact. ${ }^{1}$ The process of making this decision is customer segmentation. Not all customers have purchased identical amounts. Some have ordered more often, some have ordered more recently. Consequently, not all customers should be contacted with the same effort and expense. The cornerstone of direct marketing segmentation is recency, frequency and monetary values (RFM).

Since direct marketing segmentation is Miglautsch Marketing, Inc., W226 N555 Eastmound Drive, Ste. E, Waukesha, WI 53186-0408, USA

Tel: (262)542-5633; Fax: (262)542-2066; e-mail: john@migmar.com a science, it is important to quantify customer behaviour so that the shortand long-term effect of segmentation formulae can be tested. The purpose of RFM is to provide a simple framework for quantifying that customer behaviour. Once customers are assigned RFM behaviour scores, they can be grouped into segments and their subsequent profitability analysed. This profitability analysis then forms the basis for future customer contact frequency decisions.

\section{RFM SCORING}

The purpose of RFM scoring is to project future behaviour (driving better segmentation decisions). In order to allow projection, it is important to translate the customer behaviour into numbers which can be used through time.

Too often, direct marketers will use static customer selections. When initially building their segmentation system, they may consider their best customers to be those who have purchased more than, say, $\$ 100$. If the mailer is relatively new, this definition will degrade rapidly. The 
initial selection of $>\$ 100$ may have encompassed 20 per cent of the customer file, after a year or two, it may easily identify the top 30 per cent to 40 per cent. If the profitability of the first contact is evaluated based on the $\$ 100$ cutoff, very positive results will be seen. Given the successful segmentation experiment, the direct marketer will again use the $\$ 100$ cutoff. After substantial time elapses, that same $\$ 100$ will yield poorer results. It is not that the best customers have significantly changed, it is simply that over time, more customers will have repeat purchases and achieve the $\$ 100$ threshold.

Two common scoring methods are used to avoid the bracket creep problem, customer quintile scoring and behaviour quintile scoring.

\section{Customer quintiles}

The most common scoring method is to sort customers in descending order (best to worst). Customers are then broken into five equal groups or quintiles. The best receive a score of 5 , the worst a 1 (see Figure 1). For recency, customers are sorted by days since last purchase, the lower the number of days, the better the score. For frequency, customers are sorted by number of purchases, the higher the number of purchases, the better the score. And for monetary, customers are sorted by the amount spent. ${ }^{2}$ The higher the amount, the higher the score. Each time customers are scored, a new relative segmentation scheme would be created. It would have the advantage of quantifying customer behaviour which could be projected into the future. The relatively best customers would always fall into the 5,5,5 category. It is necessary to identify where the cutoff points fall, since they automatically change with each customer scoring.

The customer quintile method has the

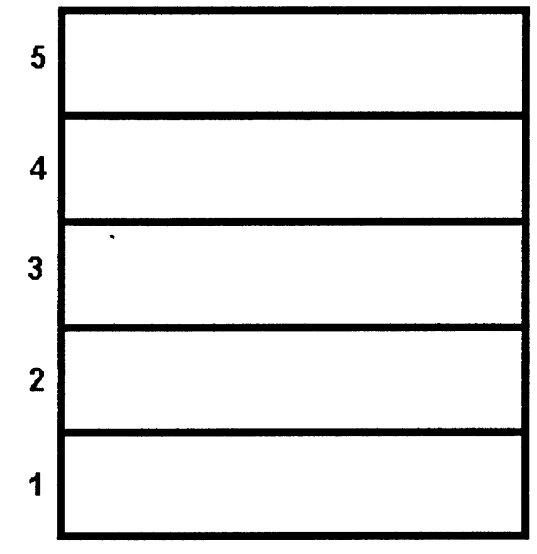

Figure 1: Equal numbers of customers in each group make analysis simpler and easier to understand

advantage of yielding equal numbers of customers in each segment. There are five equal groups for RFM, generating 125 equal size segments overall. Initial analysis would be to contact all customers, look at the performance of each individual cell (cells would have definitions like: 4,3,5 or 2,3,3) and understand how different segments of the customers perform.

With 600,000 customers there would be 4,800 in each cell. A response rate of 2 per cent would yield 96 orders giving an acceptable sample for analysis. ${ }^{3}$ With fewer than 600,000 customers, it would be highly questionable to evaluate each cell independently. Instead, the RFM would be evaluated by looking at the relative performance between the $R$ scores, the $\mathrm{F}$ scores and the $\mathrm{M}$ scores. This may not be as satisfying, but it would provide statistically significant results. Thus, a 100,000 customer mailing would have 20,000 in each group (looking only at one dimension at a time). This method extends the usefulness of RFM down to the neighbourhood of 10,000-25,000 customers. $^{4}$

The customer quintile method does encounter some scoring challenges in the 


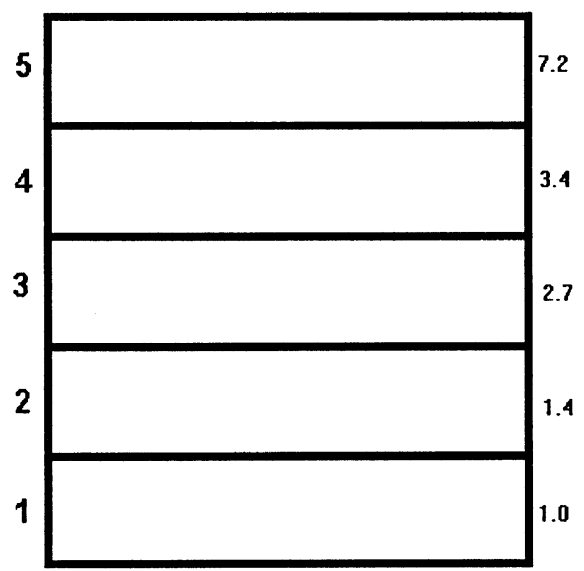

Figure 2: In this example, about $28 \%$ of the customers have only ordered once. This means over $1 / 3$ rd of the customers in score 2 have identical behaviour to score 1

area of frequency. In most direct marketing customer files, a high percentage of the customers have only ordered once. This percentage is often as high as 30 per cent to 60 per cent. If more than 20 per cent of the customers have only one purchase, then the lowest frequency group will have a purchase amount of 1 (see Figure 2). Since that group cannot hold all the customers with only one purchase, some of them will be sorted into the 2 score group. Note, their behaviour is identical to those in the 1 score, they simply spilled over. If 40 per cent of the customers had only one purchase, then both 1 and 2 score groups would have identical behaviours. If the percentage ran as high as 60 per cent (which is not that unusual) then three of the five quintiles would have the identical behaviour. Remembering the purpose of RFM, which is to quantify behaviour, this would be a less than satisfying result.

A second concern with the quintile method is its relative sensitivity. At the high end of the frequency model (see Figure 2) customers average 7.4 purchases. That is considerably more than the 1.0 purchase at the bottom and almost twice as great as the 3.4 purchases in the 4 score group. The Pareto Principle (commonly called the 80/20 rule) still applies inside the 5 score group, however. This means that there are a small number of very large customers and a larger number of relatively smaller customers who make up that 7.4 average.

As long as the segmentation scheme is primarily built for mailing purposes, this distinction is moot. Undoubtedly, the 5 and 4 groups would be mailed. If, however, the RFM model is being used to facilitate telemarketing or field sales contact, additional subsegments would be crucial to identify the super customers.

The customer quintile scoring method generates some unsatisfying results at both the top and bottom of the scale. It tends to group together customers who have vastly different buying behaviour (at the top) and arbitrarily break apart customers who have identical behaviour (at the bottom).

\section{Behaviour quintile scoring}

An alternative scoring method has been developed by John Wirth PhD. ${ }^{5}$ It also sorts customers by behaviour but instead of creating arbitrary cutoffs at a certain percent of the customers, it generates cutoffs on percentage behaviour. This method seems to overcome the sensitivity problems mentioned above. Five groups are still created, but monetary score would generate equal amounts of sales in each quintile.

Behaviour scoring has the advantage of grouping customers by similar behaviour. Since segmentation decisions are based on past customer behaviour, this allows better segmentation.

\section{Frequency}

The behaviour method does suffer from similar problems when tackling frequency 


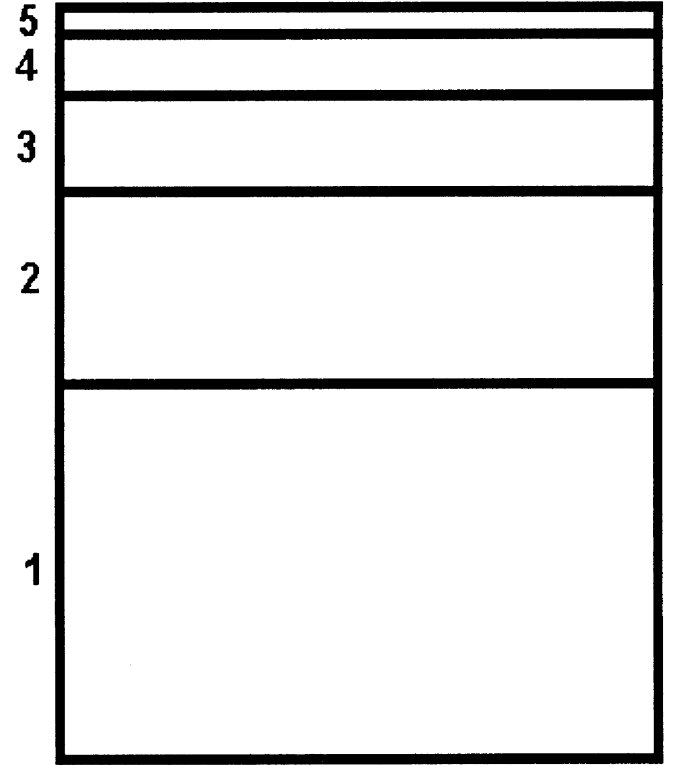

Figure 3: Though $20 \%$ of sales is in each score, the 5 score group may represent only $4 \%$ of the customers and the 1 score group may represent more than $40 \%$

score. Starting at the top of the frequency sort and subtracting each customer's frequency from total frequency, the customers who have purchased only once may not equal 20 per cent of total frequency. In that case, some of the customers who have purchased twice will be included in the 1 score group with this method.

It is also troublesome to sort customers from top to bottom in a computer generated scoring system. A special sort of file must be created and each scoring process must be accomplished uniquely. The mean scoring method, a further enhancement of the John Wirth method has been developed by Ted Miglautsch, VP Development, Miglautsch Marketing, Inc. When scoring frequency, the single purchasers are given a score of 1 . The system then averages the remaining frequencies to determine the mean. If a customer total falls below the mean, they receive a score of 2 . This process is

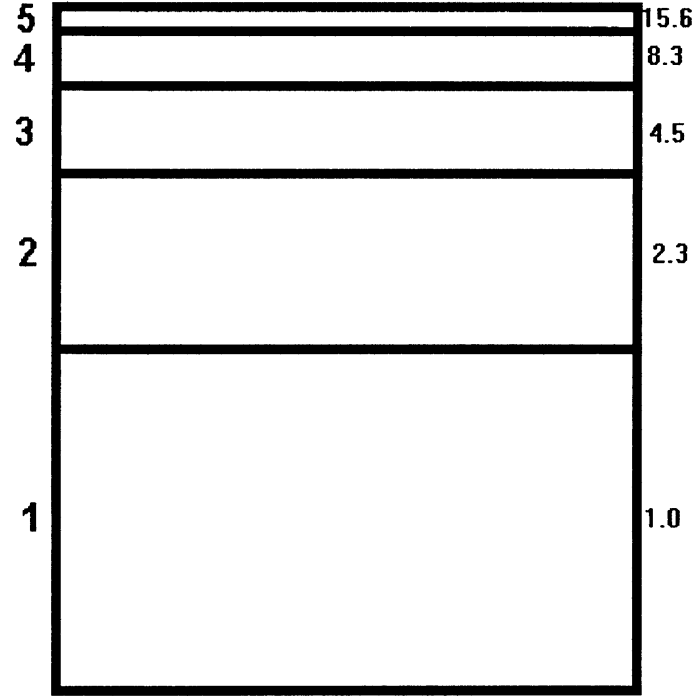

Figure 4: The mean method yields sensitivity at both top and bottom but also isolates single purchasers

repeated two more times giving quintiles of behaviour which approximate the John Wirth method, have sensitivity on both ends of the scale and allow scoring of many variables at the same time.

\section{Recency}

Since past behaviour is the best predictor of future behaviour, recency is typically considered the most powerful of the three variables. Many direct marketers make contact decisions based solely on recency. Recent customers are considered viable for a certain length of time. They are often mailed heavily in the first 12 months and increasingly less often until say 36-48 months. After that they are considered dead.

Unlike frequency and monetary, customers reset themselves. A customer reordering once in three years who purchases an average amount only once moves up in an orderly manner from a 1 
in $F \& M$ to a 2 in F\&M. But in recency, he jumps from a 1 to a 5 . Customers who order often may never have anything other than a 5 score. At the core of recency is the fact that most of the customers fall into two groups: hot and dead.

Though recency can be scored by sorting customers by days since last purchase, industry list convention suggest a more calendar-based method. 'Hotline names' typically mean purchasers within three months or 90 days. Often, marketers work very hard to make sure the most recent names are pulled out of the order processing system, sometimes within hours of the cutoff deadline. Borrowing from the Wirth method of segmenting the hottest names more finely, the most common $\mathrm{R}$ score breaks recency: 0-3 months, 4-6 months, 7-12 months, 13-24 months and 25+ months. Business-to-business direct marketers often extend these time frames since their customers can remain viable even though individuals change.

\section{Weighting}

With relational marketing databases becoming more common, most marketers can select RF\&M scores independently.

Others are not as fortunate, however, and require a single field to do the work of all three variables. The advantage of a single variable is that customers can easily be segmented by a single query on one field.

Donald R. Libey in his book, 'Libey on RFM', suggests that monetary, frequency and recency values can be added together. ${ }^{6}$ Scoring is not explicitly discussed but he does offer a formula for creating a single RFM value. His method includes adding average order and frequency per year. An example is a customer who bought 60 times, with an average order of 300 , total purchases of $\$ 18,000$ and a recency of 1 (year). Adding them all together, gives the score 18,373. This method is actually a form of weighting. Monetary value, because it is so large in comparison with recency, frequency and frequency per year overpowers the weighting.

An alternative would be simply to add the RFM scores discussed above together. The best customers would have a composite score of $15(5+5+5)$ and the worst customers would have a minimum score of $3(1+1+1)$. Many of the customers would have a score of 7 or 8 and it would be difficult to sort them. Further, the experience of decades of direct mail marketing suggests that the most recent customers are of greater value than those who have ignored more than a few repeated mailings. To enhance this composite formula, many mailers multiply $\mathrm{R} \times 5, \mathrm{~F} \times 2$ and $\mathrm{M} \times 1$. This would give the best customers a composite score of $30(5 \times 3)+$ $(5 \times 2)+(5 \times 1)$. This not only gives more power to the most recent names, it also gives a bit of a boost to frequency.

The logic behind weighting frequency is that if two customers have equal recency, spent the same amount but one ordered several times and the other only once, the more frequent buyer is much more likely to respond. If the choice were which one to mail the latest catalogue to, the choice would be the more frequent buyer.

One additional enhancement is often employed in creating a composite score. Instead of multiplying by 3,2 and 1 , substitute 9.9, 6.6 and 3.3. This yields a range of composite scores between 99 and 19.8. It preserves the approximately $3 \times$ weighting of $\mathrm{R}$, it also creates more of a 100-point scale.

\section{Life-to-date}

In general, RFM scoring is based on life-to-date totals. The author has been 
asked whether it would improve RFM scoring to shorten the time frame. The concept is that if recency is so powerful, perhaps only the recent behaviour of the past few years should be considered. An excellent suggestion but fraught with danger. The basic concept again is quantifying behaviour for the purpose of customer segmentation. It is obvious that high RFM customers are easily identified. The true challenge is to identify viable customers beyond the 12-month window. Should any of them be mailed? Certainly some should ... those who have spent a considerable sum and those who have ordered more than once (or perhaps twice). To gain this wider perspective, it requires that all available customer history be analysed.

\section{CONCLUSION}

RFM continues to provide the foundation for customer behaviour quantification. As noted, there is considerable variation in scoring methods, each has its own strengths and weaknesses. It is hoped that this discussion assists marketers in forging a solid base for measuring, understanding and executing customer segmentation.

\section{References}

1 The cost per contact varies substantially between field sales and e-mail (Internet) contact. In the latter case, customer behaviour is not so much a justification for additional contact expense as much as an indication of the depth of customer relationship. The greater the purchases, the less likely the customer will be annoyed, and the greater the risk that they are.

2 Substantial variation can be made in monetary scoring. If there is wide variation in cost of goods percent, it is better to accumulate gross margin rather than gross revenue. If there is a high percentage of returned merchandise, it would be wise to subtract returns from customer totals. In the second case, customer service costs are also often subtracted from the total.

3 Though each cell would have the same quantity, response rate would vary. It is beyond the scope of this paper to explore confidence interval calculations at the cell and the mailing level.

42,000 customers in each cell at an average of 2 per cent response would generate 40 responses. Confidence level would be \pm 10 per cent. Most mailers would consider this minimally projectable. It is important to note, however, that the 2 per cent would not be consistent across cells.

5 John Wirth did his course work in aeronautical engineering. He is the founder of Woodworker's Supply of New Mexico, a leading hand tool catalogue and retailer.

6 Libey, D. R. 'Libey on RFM', http://e-RFM.com. 